\title{
Um modelo computacional baseado em redes semânticas para análises em vereditos jurídicos
}

\author{
Carvalho, J. D. S. G. ${ }^{1}$; Nascimento, J. O. do ${ }^{1}$; Moret, M. A A $^{1,2}$ \\ 1 Programa de Modelagem Computacional, CIMATEC, Salvador, BA, Brasil. \\ 2 Universidade do Estado da Bahia, Salvador, Bahia, Brasil.
}

e-mail: joanadarc.galvao@gmail.com, jeffersonascimento@gmail.com

\begin{abstract}
Resumo
No tocante aos sistemas complexos, pode-se encontrar várias estruturas, ou sejam características, comportamento complicado, complexo, decorrente de uma forma simples, assim pode-se concluir que é um sintoma clássico de um Sistema complexo. Um texto pode parecer uma atividade simples, mas quando temos uma sentença(veredito) judicial, teremos institutos que obedecem às uniões significantes ou vocábulos, e as suas influências ocorrem por meio das sentenças do texto. Surge assim, a ideia de apresentar um sistema complexo acurado através da Teoria de Redes, no caso as redes semânticas, fundamentadas em analisar as sentenças judiciais, chamados também de vereditos, pois os magistrados detêm dessas prerrogativas de julgar e sentenciar em lides judiciais. A área jurídica pesquisa foi a trabalhista com julgados entre 2013 a 2018 pelo Tribunal Regional do Trabalho da 5a região. Expomos o método de construção das redes semânticas inicialmente com a preparação dos dados, organizando as sentenças, para aplicação da modelagem computacional com a ajuda de softwares para construção e visualização das redes. As redes semânticas podem apresentar características muito boas para determinados tipos de problemas, tais como de reconhecimento de padrões, e de tratamento de linguagem natural. Os índices estatísticos ligados a teoria de redes complexas e as medidas de centralidades de grau e proximidade pertencentes às redes sociais são medidos e apresentados. Geralmente pelos padrões que são comparecidos, as redes semânticas por sua origem se distinguem apresentando o evento Small-World e há indicios de que também sejam redes Scale Free.
\end{abstract}

Palavras-chave: Sistemas Complexos, Redes Sociais e Complexas, Redes Semânticas, Vereditos Judiciais.

\begin{abstract}
With regard to complex systems, one can find several structures, that is, complex, complicated behavior, complex, arising in a simple way, so it can be concluded that it is a classic symptom of a complex System. A text may seem like a simple activity, but when we have a judicial verdict, we will have institutes that obey significant unions or vocabulary, and their influences occur through the sentences of the text. Thus, the idea of presenting a complex system through Network Theory, in the case of semantic networks, based on analyzing judicial sentences, also called verdicts, since magistrates have these prerogatives to judge and sentence in judicial litigation. The legal research area was labor with judged between 2013 to 2018 by the Regional Labor Court of the 5th region. We present the method of constructing the semantic networks initially with the preparation of the data, organizing the sentences, for application of the computational modeling with the help of software for construction and visualization of the networks. Semantic networks may have very good characteristics for certain types of problems, such as pattern recognition, and natural language processing. The statistical indexes linked to complex network theory and the measures of degree and proximity centrality belonging to social networks are measured and presented. Generally by the standards that are presented, the semantic networks by their origin are distinguished presenting the Small-World event and there are indications that they are also Scale Free networks.
\end{abstract}

Keywords: Complex Systems, Social and Complex Networks, Semantic Networks, Judicial Verdicts. 


\section{Introdução}

A importância desse artigo se dá quando se devem tomar decisões complicadas, difíceis, ou que poderiam ter mais de um resultado nas sentenças judiciais. O objetivo desse artigo é tratar dos conceitos e aplicações em torno de uma análise dos padrões estudados em seus sistemas complexos, através da Teoria de Redes.

O mundo dos sistemas complexos é o mundo das transições de fase, das mudanças bruscas de comportamento ou das bifurcações, da manifestação de ordem em métodos caóticos, da ocorrência de processos intensos como a explosão de uma supernova ou a extinção de uma espécie. Estes processos estão invariavelmente associados a modelos matemáticos não-lineares, esta exposição está organizada tendo como ponto de partida as relações entre o linear e o não-linear, tentando mostrar a diversidade de fenómenos que podem surgir nos sistemas complexos [1].

A averiguação dos sistemas complexos acontece com o formalismo matemático de sistemas dinâmicos, com equações diferenciais, equações por meio de diferenças, mapas logísticos, autômatos celulares, redes dinâmicas (teoria de redes), etc. (ibidem), cuja ferramenta fundamental é a utilização de recursos computacionais [2]. Tem se o entendimento que a teoria de Redes Complexas e Redes sociais cartear-se a formas de analisarmos sistemas complexos [2].

Inspirados em Nascimento et al. [1] e Nascimento, Pereira e Moret [2], o objetivo da pesquisa é exibir um sistema complexo, através da Teoria de Redes (redes semânticas baseadas em sentenças judiciais). As análises efetivadas são aplicadas em sentenças proferidas pela $5^{\circ}$ Tribunal Regional do Trabalho, em Salvador, BA, oriundas de informações disponíveis publicamente e, que os solicitantes de serviços advocatícios tenham escolhido pelo escritório partícipe da pesquisa).

Organizou-se o presente trabalho em cinco partes. Na segunda serão apresentados os materiais e métodos empregados na construção da rede semântica. Na terceira parte, apresentaremos os resultados achados com as análises das redes semânticas. Na quarta parte, as considerações finais. Na quinta e última parte, as referências empregadas na preparação deste artigo.

\section{Materiais e Métodos}

A base de dados utilizada está disponível de forma pública, que correspondem em sentenças proferidas pela $5^{\circ}$ Tribunal Regional do Trabalho, na capital da Bahia, Salvador. De acordo com a Teoria de Redes, para representarmos uma rede semântica, deve-se observar os princípios da Teoria dos Grafos. Um grafo $G=(V, E)$ é em uma estrutura matemática composta por dois conjuntos: o conjunto de vértices (V), que é finito e não vazio) e, o conjunto das arestas $(E)$, as relações binárias sobre $V$ ) [3]. Os índices estatísticos pertencentes à Teoria dos Grafos que são utilizados nas redes semânticas deste artigo são os descritos na Tabela:

Tabela 1: índices pertencentes às redes complexas. Fonte: Nascimento, Pereira e Moret [4].

\begin{tabular}{c|c|c}
\hline$n$ & Número de Vértices & $n=|V|$ \\
\hline$m$ & Número de Arestas & $m=|E|$ \\
\hline$\langle k\rangle$ & Grau Médio do Vértice & $\langle k\rangle=\frac{1}{n} \sum_{i}^{n} k_{i}$ \\
\hline$\Delta$ & Densidade da Rede & $\Delta=\frac{m}{n(n-1) / 2}$ \\
\hline$C_{v}$, & Coeficiente de & $C_{V}=\frac{2 E_{v}}{k_{v}\left(k_{v}-1\right)}$ \\
\hline$C_{w s}$ & Aglomeração do Vértice & $C_{W S}=\frac{1}{N} \sum_{V=1}^{N} C_{V}$ \\
\hline$L$ & Caglomeração da Rede & $L=\frac{1}{n(n-1)} \sum_{i \neq j} d_{i j}$ \\
\hline$D$ & Diâmetro da Rede & $D=\max \left(d_{i j}\right)$ \\
\hline
\end{tabular}

Utilizamos as obras de Pereira et al. [5], Nascimento et al. [1], Nascimento, Pereira e Moret [2] e Nascimento et al. [6] para demonstrarmos o método de construção da rede semântica baseada vereditos (sentenças) judiciais, desde o pré-processamento das palavras até a utilização de softwares. 


\section{Resultados}

Realizamos a construção e as análises de duas redes semânticas originadas a partir de dois vereditos jurídicos distinto escolhidos de forma aleatória da nossa base de dados. Desta forma, apresentamos a Figura 1 a rede semântica baseada no

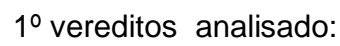

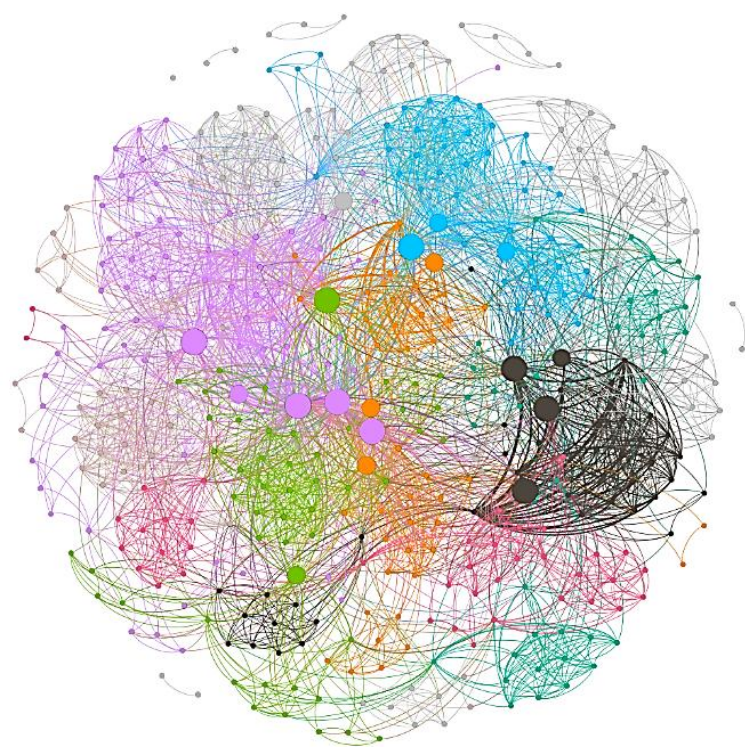

Figura 1: Rede semântica complexa baseada no $1^{\circ}$ veredito jurídico da base de dados da pesquisa. Fonte: Dos autores.

A rede semântica da Figura 1 apresenta os tamanhos dos vértices proporcionais aos valores medidos da centralidade de grau (medida de importância de um vértice por meio das conexões com vértices vizinhos). Destacando que os vértices com maiores tamanhos e próximos à região central, correspondem aos que se destacam pela medida de Centralidade de Grau. Na Tabela 2 estão disponíveis os índices de redes complexas conforme a Tabela 1, verificados para a rede semântica da Figura 1:

Tabela 2: índices pertencentes às redes complexas. Fonte: Dos autores

\begin{tabular}{|c|c|c|c|c|}
\hline Rede & Índices & Valor & Índices & Valor \\
\hline \multirow{4}{*}{$\begin{array}{l}\text { Veredito } \\
\text { Jurídico } 1\end{array}$} & $n=|V|$ & 441 & $\begin{array}{c}\text { Quantidade } \\
\text { de } \\
\text { componentes }\end{array}$ & 7 \\
\hline & $\boldsymbol{m}=|E|$ & 4016 & $\langle k\rangle$ & 18,62 \\
\hline & $\Delta$ & 0,042 & $c_{W s}$ & 0,89 \\
\hline & $L$ & 2,541 & $D$ & 4 \\
\hline \multirow{2}{*}{$\begin{array}{c}\text { Veredito } \\
\text { Jurídico } 1 \text { - } \\
\text { Maior } \\
\text { Componente }\end{array}$} & $n=|V|$ & 427 & $\begin{array}{c}\text { Maior } \\
\text { Componente } \\
(\%)\end{array}$ & 96,83 \\
\hline & $m=|E|$ & 4094 & $\langle k\rangle$ & 19,17 \\
\hline
\end{tabular}

\begin{tabular}{c|c|c|c|c}
\hline & $\boldsymbol{\Delta}$ & 0,42 & $\boldsymbol{C}_{w s}$ & 0,89 \\
\cline { 2 - 5 } & $\boldsymbol{L}$ & 2,542 & $\boldsymbol{D}$ & 4 \\
\hline \multirow{4}{*}{$\begin{array}{c}\text { Rede } \\
\text { aleatória } \\
\text { equivalente. }\end{array}$} & $\boldsymbol{n}=|\boldsymbol{V}|$ & 427 & $\begin{array}{c}\text { Maior } \\
\text { Componente } \\
(\%)\end{array}$ & 100 \\
\cline { 2 - 5 } & $\boldsymbol{m}=|\boldsymbol{E}|$ & 4056 & $\langle\boldsymbol{k}\rangle$ & 19,17 \\
\cline { 2 - 5 } & $\boldsymbol{\Delta}$ & 0,04 & $\boldsymbol{C}_{w s}$ & 0,011 \\
\cline { 2 - 5 } & $\boldsymbol{L}$ & 2,511 & $\boldsymbol{D}$ & 4 \\
\hline
\end{tabular}

A rede semântica da Figura 2 é a correspondente ao $2^{\circ}$ veredito jurídico utilizado na presente pesquisa, para podermos comparar com os resultados do $1^{\circ}$.

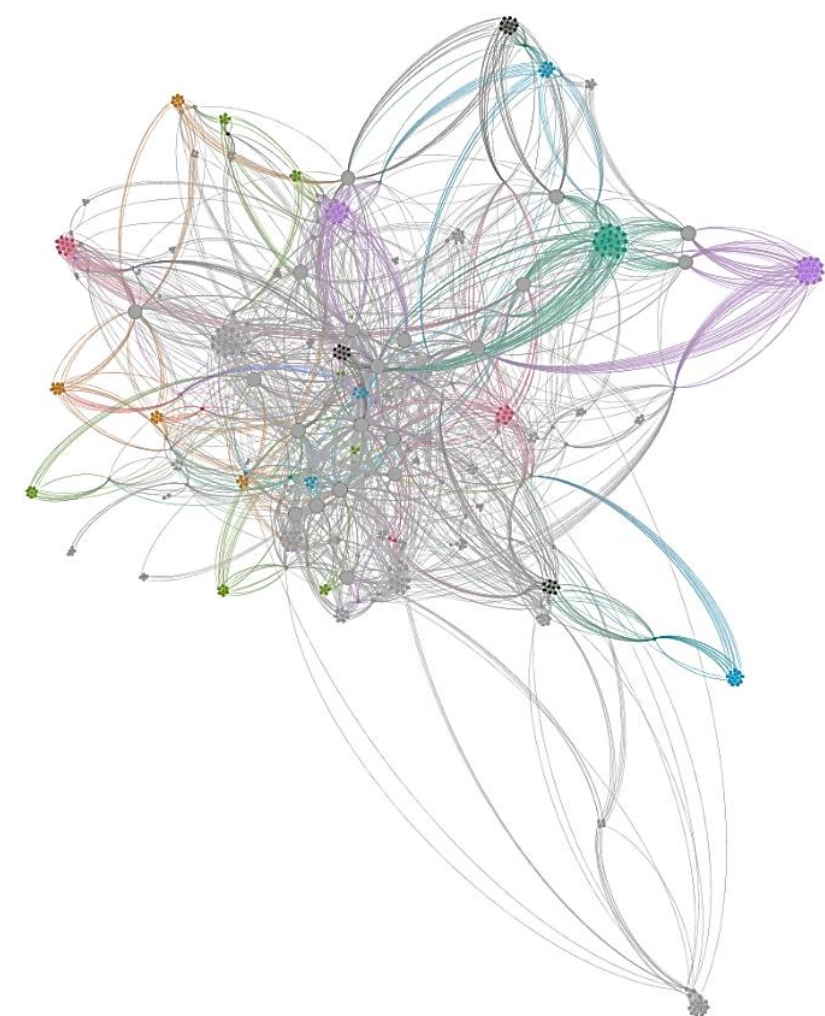

Figura 2: Rede semântica complexa baseada no $2^{\circ}$ veredito jurídico da base de dados da pesquisa. Fonte: Dos autores.

Na Tabela 3 estão disponíveis os índices de redes complexas apresentados na Tabela 1, verificados para a rede semântica da Figura 2:

Tabela 3: índices pertencentes às redes complexas. Fonte: Dos autores.

\begin{tabular}{c|c|c|c|c}
\hline Rede & Índices & Valor & Índices & Valor \\
\hline \multirow{2}{*}{$\begin{array}{c}\text { Veredito } \\
\text { Juridico 2 }\end{array}$} & $\boldsymbol{n}=|\boldsymbol{V}|$ & 547 & $\begin{array}{c}\text { Quantidade } \\
\text { de } \\
\text { componentes }\end{array}$ & 8 \\
\cline { 2 - 5 } & $\boldsymbol{m}=|\boldsymbol{E}|$ & 5245 & $\langle\boldsymbol{k}\rangle$ & 19,17 \\
\cline { 2 - 5 } & $\boldsymbol{L}$ & 0,035 & $\boldsymbol{C}_{w s}$ & 0,899 \\
\cline { 2 - 5 } & $\boldsymbol{L}$ & 2,597 & $\boldsymbol{D}$ & 6 \\
\hline & $\boldsymbol{n}=|\boldsymbol{V}|$ & 533 & $\begin{array}{c}\text { Maior } \\
\text { Componente }\end{array}$ & 97,44 \\
\hline
\end{tabular}




\begin{tabular}{|c|c|c|c|c|}
\hline \multirow{4}{*}{$\begin{array}{c}\text { Veredito } \\
\text { Jurídico } 1 \text { - } \\
\text { Maior } \\
\text { Componente }\end{array}$} & & & (\%) & \\
\hline & $\boldsymbol{m}=|E|$ & 5229 & $\langle k\rangle$ & $\begin{array}{c}19,62 \\
1\end{array}$ \\
\hline & $\Delta$ & 0,037 & $C_{w s}$ & 0,897 \\
\hline & $L$ & 2,598 & $D$ & 6 \\
\hline \multirow{4}{*}{$\begin{array}{c}\text { Rede } \\
\text { aleatória } \\
\text { equivalente. }\end{array}$} & $n=|V|$ & 533 & $\begin{array}{c}\text { Maior } \\
\text { Componente } \\
(\%)\end{array}$ & 100 \\
\hline & $m=|E|$ & 5268 & $\langle\boldsymbol{k}\rangle$ & 19,62 \\
\hline & $\Delta$ & 0,031 & $C_{w s}$ & 0,003 \\
\hline & $L$ & 2,666 & $D$ & 6 \\
\hline
\end{tabular}

A rede semântica da Figura 1 foi construída de modo a apresentar em seu centro os vértices com maiores números de conexões na rede. Como já mencionado, os vértices mais conectados nela apresentam maiores importâncias por este fato (medida de centralidade de grau). Tanto na Figura 1 quando na Figura 2 utilizamos os algoritmos Force Atlas 2, Não sobrepor e o OpenOrd. Na rede semântica da Figura 2 os vértices com maiores valores de centralidade de grau não estão localizados na região central. Porém continuam diferenciados pelo seus respetivos tamanhos, conforme 0 valor da supracitada centralidade.

Nas Tabela 2 e Tabela 3, apresentamos a comparação entre os índices calculados para a rede completa, a maior componente dela e a rede aleatória equivalente para cada uma. Desta forma, seguimos os nortes fornecidos por Watts e Strogatz [7], com o intuito de podemos realizar uma comparação entre a maior componente de cada rede e as suas respetivas redes aleatórias equivalentes. Desta forma, pudemos argumentar sobre o fenômeno Small-World.

Assim, percebemos que as redes apresentam valores aproximadamente iguais para o caminho mínimo médio (L). Já os coeficientes de aglomerações médios $\left(\mathrm{C}_{\mathrm{ws}}\right)$ das redes dos vereditos jurídicos apresentaram valores elevados em comparação com os coeficientes de aglomeração das respectivas redes aleatórias equivalentes. Pelo referido método, há uma sugestão de que as redes semânticas apresentam o referido fenômeno Small-Word: elevada aglomeração local e baixas geodésicas [7].
Realizamos também a distribuição de graus das redes semânticas analisadas, conforme Figura 3 e Figura 4 a seguir:

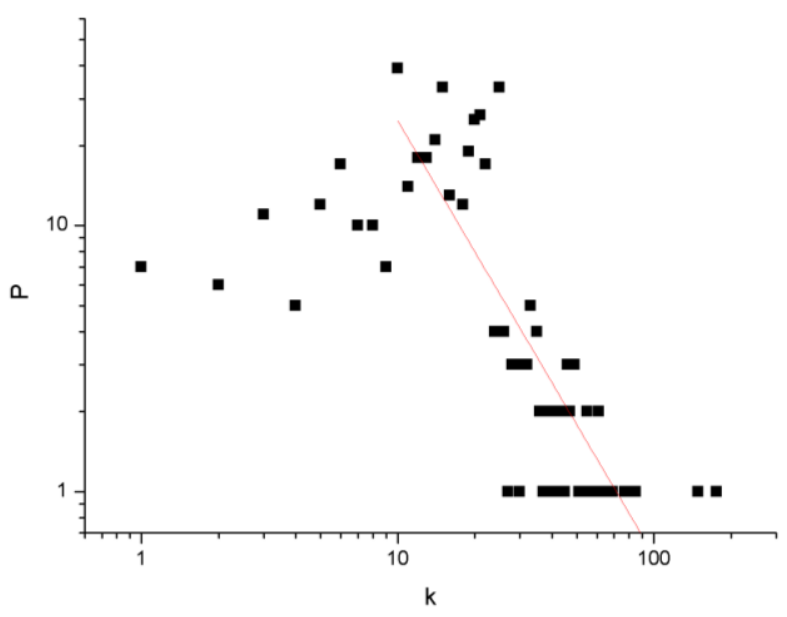

Figura 3: Distribuição de graus da rede semântica baseada no parecer jurídico 1 analisado (com $\gamma=1,9559$ e o ajuste $\left.R^{2}=0,72041\right)$. Fonte: Dos autores.

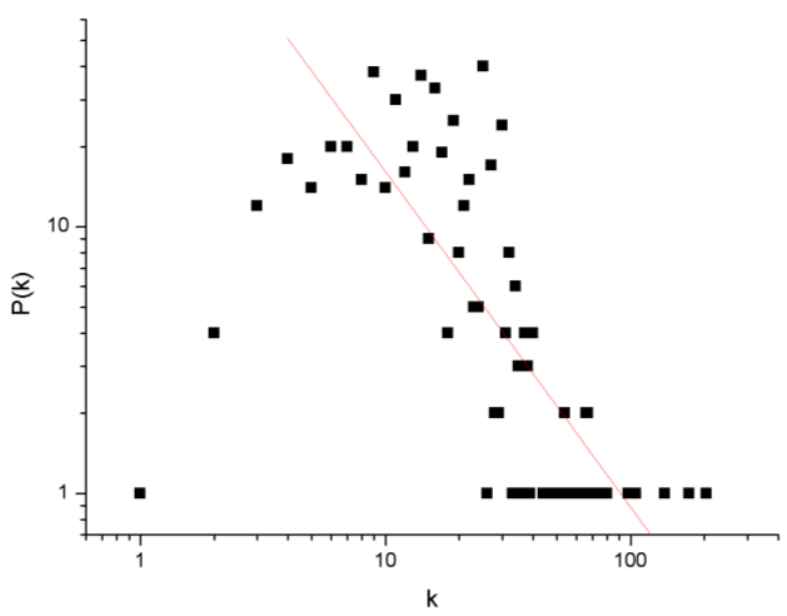

Figura 4: Distribuição de graus da rede semântica baseada no parecer jurídico 2 analisado (com $\gamma=1,257$ e o ajuste $\left.R^{2}=0,71102\right)$. Fonte: Dos autores.

Ao observamos as figuras anteriores correspondentes às distribuições de graus, há um forte indicativo de que a topologia das redes semânticas dos pareceres jurídicos possam seguir uma lei de potência da forma $F(k)=P(k) \sim k^{-\gamma}$, o que corresponde a uma característica de redes Scale Free (redes livres de escala) [8]. Uma rede pode apresentar o fenômeno Small-World e ser Scale Free concomitantemente. Ambas as características não são excludentes entre si. A presença de hubs ocorre nessas redes e, elencamos os quarenta primeiros vértices mais conectados para ambas as redes analisadas, com fortes indícios de corresponderem a hubs, conforme a tabela 4 e tabela 5: 
Tabela 4: Centralidade de Grau dos quarenta vértices mais conectados da rede semântica baseada no parecer jurídico 1 analisado. Fonte: Dos autores.

\begin{tabular}{|c|c|c|c|c|c|}
\hline & $\begin{array}{l}\text { Vértices } \\
\text { (V) }\end{array}$ & $k$ & & $\begin{array}{l}\text { Vértices } \\
\text { (V) }\end{array}$ & $k$ \\
\hline 1 & Não & 176 & 21 & Pagamento & 46 \\
\hline 2 & Ser & 149 & 22 & Forma & 46 \\
\hline 3 & Poder & 85 & 23 & Observar & 46 \\
\hline 4 & DANO MORAL & 77 & 24 & Passar & 45 \\
\hline 5 & Reclamante & 69 & 25 & Evitar & 44 \\
\hline 6 & Haver & 63 & 26 & Empregado & 44 \\
\hline 7 & Reclamar & 62 & 27 & Objeto & 42 \\
\hline 8 & Pedido & 61 & 28 & Recolhimento & 42 \\
\hline 9 & Dever & 61 & 29 & $\begin{array}{c}\text { Administração } \\
\text { pública }\end{array}$ & 41 \\
\hline 10 & Ter & 58 & 30 & Modo & 40 \\
\hline 11 & Empresa & 55 & 31 & Sofrer & 39 \\
\hline 12 & Parte autora & 55 & 32 & Através & 38 \\
\hline 13 & Incidir & 54 & 33 & Indenização & 38 \\
\hline 14 & Comprovar & 53 & 34 & Demonstrar & 37 \\
\hline 15 & Ficar & 51 & 35 & Ação & 36 \\
\hline 16 & Prova & 49 & 36 & Autor & 36 \\
\hline 17 & Responsabilidade & 49 & 37 & Ensejar & 35 \\
\hline 18 & Relação & 49 & 38 & Presunção & 35 \\
\hline 19 & Fixar & 47 & 39 & Lesão & 35 \\
\hline 20 & Valor & 47 & 40 & Estar & 35 \\
\hline
\end{tabular}

Tabela 5: Centralidade de Grau dos quarenta vértices mais conectados da rede semântica baseada no parecer jurídico 2 analisado. Fonte: Dos autores.

\begin{tabular}{c|c|c|c|c|c}
\hline \multicolumn{2}{c|}{$\begin{array}{c}\text { Vértices } \\
\text { (V) }\end{array}$} & $\boldsymbol{k}$ & \multicolumn{2}{c|}{$\begin{array}{c}\text { Vértices } \\
\text { (V) }\end{array}$} & $\boldsymbol{k}$ \\
\hline 1 & Não & 204 & 21 & Prazo & 54 \\
\hline 2 & Ser & 173 & 22 & Obreiro & 54 \\
\hline 3 & Reclamar & 138 & 23 & São & 53 \\
\hline 4 & Dever & 105 & 24 & Comprovar & 48 \\
\hline 5 & Empregado & 104 & 25 & Rescisão indireta & 47 \\
\hline 6 & Reclamante & 98 & 26 & Anotação & 46 \\
\hline 7 & Parte & 80 & 27 & Causar & 44 \\
\hline 8 & Estar & 78 & 28 & Inicial & 40 \\
\hline 9 & Haver & 75 & 29 & Deferir & 40 \\
\hline 10 & Pagamento & 70 & 30 & Sentido & 40 \\
\hline 11 & Posto & 67 & 31 & Contratar & 40 \\
\hline 12 & Atraso & 67 & 32 & Condição & 39 \\
\hline 13 & Vez & 66 & 33 & Imputar & 38 \\
\hline 14 & Nunca & 66 & 34 & $\begin{array}{c}\text { Responsabilidade } \\
\text { subsidiaria }\end{array}$ & 38 \\
\hline 15 & Efetuar & 64 & 35 & $\begin{array}{c}\text { Honorários } \\
\text { advocatícios }\end{array}$ & 38 \\
\hline 16 & Além & 61 & 36 & Decidir & 37 \\
\hline 17 & $\begin{array}{c}\text { Contrato de } \\
\text { trabalho }\end{array}$ & 60 & 37 & Fazer & 37 \\
\hline 18 & Trabalho & 58 & 38 & Lei & 37 \\
\hline 19 & $\begin{array}{c}\text { Ambiente de } \\
\text { trabalho }\end{array}$ & 57 & 39 & Dizer & 37 \\
\hline 20 & Proceder & 56 & & $\begin{array}{c}\text { Sindicato da } \\
\text { categoria }\end{array}$ & \\
& profissional & 36 \\
\hline & & 40 & &
\end{tabular}

Conforme os dados elencados nas tabelas anteriores, percebemos que os vértices com maiores valores de centralidade de Grau, estão indicando nortes referentes as principais características jurídicas dos documentos. Logo, há uma forte tendência de que as redes semânticas dos pareces jurídicos, pela medida de centralidade de grau, aponte na direção das características fundamentais de tais documentos. Há também indicadores de que, por estes vértices, que norteiam a construção do que chamaremos aqui de "vocabulário do parecer jurídico", possamos apontar características cognitivas do redator do documento. Tais encaminhamentos correspondem aos próximos passos das pesquisas em andamentos.

\section{Considerações finais}

A presente pesquisa em caráter inicial, apresentou a possibilidade de análises de vereditos jurídicos por meio de redes sociais e complexos, com a construção e análise de redes semânticas. Os resultados indicaram que as redes apresentam 0 fenômeno Small-World e redes Scale Free. Os valores dos coeficientes angulares estão coerentes com a literatura científica recente para redes semânticas, como em NASCIMENTO et al. [1], Nascimento, Pereira e Moret [2], Fadigas et al. [9] e Seyed-allaei et al. [10].

Em relação às análises dos modelos computacionais resultantes nas redes semânticas para a área jurídica, pudemos perceber que apontaram na direção de uma análise cognitiva e/ou pela difusão da informação nos textos que constituem a base de dados utilizadas. Desta forma, as pesquisas continuarão para que possamos expandir os resultados para maiores contribuições para área em foco, por meio da modelagem computacional.

\section{Agradecimentos}

Marcelo A. Moret agradece ao CNPq pelo suporte financeiro parcial oriundo de sua bolsa de Produtividade em Pesquisa (No. 305291/2018-1).

\section{Referências}

[1] NASCIMENTO, J. O. do; PEREIRA, H. B. B. ; CUNHA, M. V. ; MORET, M. A. Sistemas complexos e 
Ciências das redes: redes semânticas baseadas em abstracts e keywords do Ensino de Física Nacional. REVISTA CEREUS, v. 11, p. 161-183, 2019.

[2] NASCIMENTO, J. O. do; PEREIRA, H. B. B.; MORET, M. A Grafos e Teoria de Redes: uma análise do Ensino de Física Brasileiro no período 1972-2006 por meio de cliques de palavras-chave. REVISTA CEREUS, v. 10, p. 315-339, 2018.

[3] GROSS, J. L.; YELLEN, J. Graph theory and its applications. CRC press, Boca Raton FL USA 2005.

[4] NASCIMENTO, J. O. do ; PEREIRA, H. B. B. ; MORET, M. A. Redes Complexas e Sociais como ferramentas para o estudo de um sistema complexo baseado em keywords de produções científicas. In: IX Encontro Científico de Física Aplicada, 2018, São Paulo. Blucher Physics Proceedings. São Paulo: Editora Blucher, 2018. v. 4. p. 69-73.

[5] PEREIRA, H. B. B. ; FADIGAS, Inácio de Sousa; SENNA, V.; MORET, M. A. Semantic Networks based on Titles of Scientific Papers. Physica A (Print), v. 390, p. 1192-1197, 2011.
[6] NASCIMENTO, J. O. do; MONTEIRO, R. L. S.; MOREIRA, D. M.; MORET, Marcelo A.; PEREIRA, H. B. B. Semantic networks of keywords from Brazilian dissertations on physics teaching. Discontinuity, Nonlinearity and Complexity, 2018.

[7] WATTS, Duncan J.; STROGATZ, Steven H. Collective dynamics of 'small-world'networks. Nature, v. 393, n. 6684, p. 440-442, 1998.

[8] BARABÁSI, A. L. E ALBERT, R.. "Emergence of scaling in random networks". Science, n. 286, pp. 509512, 1999.

[9] FADIGAS, I. S., CASAS, T. H. P., SENNA, V., MORET, M. A, PEREIRA, H. B. B. "Análise de redes semânticas baseada em títulos de artigos de periódicos científicos: o caso dos periódicos de divulgação em educação matemática". Educação Matemática Pesquisa, 11, p. 167-193, 2009.

[10] SEYED-ALLAEI, H., Bianconi \& M. Marsili, G. Scale-free networks with an exponent less than two. Phys. Rev. E. 73, 046113, 2006. 